\title{
Energetic oxygen atoms in the polar geocorona
}

\author{
V. I. Shematovich, ${ }^{1}$ D. V. Bisikalo, ${ }^{1}$ and J.-C. Gérard ${ }^{2}$ \\ Received 30 April 2006; revised 26 June 2006; accepted 7 July 2006; published 4 October 2006.
}

[1] The role of the auroral sources induced by the electron and proton precipitation in the formation of the hot oxygen corona in the polar upper atmosphere is studied. It is found that both electron precipitation through exothermic chemistry and proton precipitation through atmospheric sputtering significantly contribute to the population of the hot oxygen geocorona. It is also found that only atmospheric sputtering results in the formation of the escape flux of energetic oxygen atoms, providing an important source of heavy atoms for the magnetosphere. The exothermic chemistry induced by the electron precipitation and/or by the absorption of the solar UV radiation is operating continuously in the polar upper atmosphere and results in a steady population of the very near-Earth environment by suprathermal oxygen atoms with energies below a few eV. By contrast, atmospheric sputtering by magnetospheric protons provides a more variable contribution, strongly coupled with the cusp region. It produces the more energetic oxygen atoms that populate the external regions of the hot oxygen geocorona. The results of calculations are in a good agreement with the analysis of the low-latitude perigee Low Energy Neutral Atom (LENA) images showing that the instrument signal consists of low to medium energy $(5-30 \mathrm{eV})$ oxygen atoms produced in and near the cusp region. The more energetic $(>30 \mathrm{eV})$ fraction of energetic oxygen atoms produced by the ioninduced atmospheric sputtering could be responsible for the energetic neutrals observed by the instrument far away from the cusp or oval regions. The total escape flux of oxygen atoms associated with atmospheric sputtering by protons is found about $8 \times 10^{23} \mathrm{~s}^{-1}$; therefore this mechanism may provide a substantial contribution to the magnetospheric oxygen population.

Citation: Shematovich, V. I., D. V. Bisikalo, and J.-C. Gérard (2006), Energetic oxygen atoms in the polar geocorona, J. Geophys. Res., 111, A10301, doi:10.1029/2006JA011823.

\section{Introduction}

[2] The hot oxygen geocorona has generated recent studies for several reasons. It is a source region of energetic neutral atoms (ENA), it contributes to the maintenance of the nighttime ionosphere, it plays a role in the formation of the escape flux of neutral atoms, and it controls the energetic ion populations in the thermosphere.

[3] The Low Energy Neutral Atom (LENA) instrument on board the IMAGE spacecraft has provided the first global observations of low-energy ENA emissions in the terrestrial atmosphere. Analysis of ENA data from LENA shows that the Earth's upper atmosphere is a copious emitter of energetic neutral atoms $(<300 \mathrm{eV})$ under all conditions [Wilson et al., 2003; Wilson and Moore, 2005]. Two different patterns were found in the ENA images, probably corresponding to different sources of these hot atoms. When solar activity is low, the observed emissions are concentrated close to the Earth and are presumably the high-energy tail of the hot oxygen geo-

\footnotetext{
${ }^{1}$ Institute of Astronomy, Russian Academy of Sciences, Moscow, Russia.

${ }^{2}$ Laboratoire de Physique Atmosphérique et Planétaire, Université de Liège, Liège, Belgium.

Copyright 2006 by the American Geophysical Union. 0148-0227/06/2006JA011823\$09.00
}

corona, with energies $<2 \mathrm{eV}$. This source exhibits little time variability. When the activity increases, the relative abundance of the higher-energy neutrals increases and the emissions can be seen farther from the Earth. This source is localized in the auroral zone and produces more energetic particles with a high degree of time variability.

[4] Three main processes have been identified as sources of ENAs near the Earth that would be observable with the LENA instrument (energies above $10 \mathrm{eV}$ and less than $300 \mathrm{eV}$ in the spacecraft frame [Moore et al., 2000]). The energetics of such processes is initiated by the absorption of the solar ultraviolet radiation and/or of precipitation of the high-energy magnetospheric ions. The first of these processes is the energetization of ionospheric ions followed by the charge exchange with neutral atoms in the upper thermosphere or geocorona [Yau and André, 1997; Gardner and Schunk, 2004, 2005]. Auroral energetization of light and heavy ionospheric ions $\left(\mathrm{H}^{+}, \mathrm{He}^{+}, \mathrm{N}^{+}, \mathrm{O}^{+}, \mathrm{NO}^{+}, \mathrm{O}_{2}^{+}, \mathrm{N}_{2}^{+}\right)$and their outflow are controlled by the different acceleration mechanisms such as Joule heating, hot magnetospheric ions and electrons, auroral ion transverse heating and parallel acceleration, electromagnetic wave turbulence, and centrifugal acceleration [cf. Schunk and Nagy, 2000]. Precipitation of high-energy magnetospheric ions results in the production of the low-energy ENAs by two routes. The first is the so- 
called ENA mirroring process [Galand and Richmond, 1999; Gérard et al., 2000] where precipitating ions are turned upward through a set of charge exchange and charge stripping collisions, with energy less than the ion's initial energy. The second route is a so-called atmospheric sputtering [Johnson, 1994] where energetic protons precipitating from the magnetosphere [Shematovich et al., 2005] or oxygen ions from the ring current [Ishimoto et al., 1992; Bisikalo et al., 1995] eject low-energy oxygen ions from the thermosphere. The third process for producing low-energy ENA near the Earth consists of a set of exothermic chemical reactions in which oxygen atoms are formed with suprathermal energies up to $5 \mathrm{eV}$ [Richards et al., 1994; Hickey et al., 1995; Gérard et al., 1995]. These low-energy oxygen atoms can be detected by the LENA instrument when their energy in spacecraft frame is boosted by the ram effect. The first two ENA production processes are closely associated with the auroral oval while the last one occurs over the whole sunlit hemisphere.

[5] In this paper we provide estimates of the importance of the above mentioned auroral sources and their contribution to the upward fluxes of hot oxygen, i.e., low-energy ENA fluxes.

\section{Sources of Energetic ( $E>1 \mathrm{eV})$ Oxygen Atoms}

[6] Suprathermal atoms are generally considered to be particles with kinetic energies above 5-10 $T$, where $T$ is the temperature of an ambient atmospheric gas. Suprathermal particles are produced in various physical and chemical processes whose products have an excess kinetic energy owing to exothermic chemistry induced by photons and electrons and atmospheric sputtering by the solar wind, magnetospheric or pick-up ions. Usually, these suprathermal atoms of nonthermal origin together with atoms from the suprathermal tail of the local Maxwellian distribution for the ambient atmospheric gas are called as hot atoms. Finally, atoms ejected by the atmospheric sputtering or formed in the charge exchange with high-energy ions and reaching excess kinetic energies up to hundred $\mathrm{eV}$ are called as energetic atoms for a convenience of interpretation of the ENA instrument data.

\subsection{Auroral Electron Precipitation and Induced Exothermic Ion-Molecular Chemistry}

[7] Exothermic ion-molecular reactions and especially dissociative recombination of molecular oxygen ions are an important source of suprathermal atomic oxygen in the upper atmospheres of the terrestrial planets (see, in particular, Shematovich et al. [1994, 1999], Hickey et al. [1995], and Fox [2005]). An extended set of exothermic reactions relevant to the formation of hot atomic oxygen in the Earth's upper atmosphere was given and analyzed by Richards et al. [1994], Hickey et al. [1995], and Gérard et al. [1995]. This set of exothermic chemical reactions was used in the present study with the following update of the $\mathrm{O}_{2}^{+}$dissociative recombination channels and their branching ratios:

$$
\mathrm{O}_{2}^{+}+e \rightarrow\left\{\begin{array}{l}
\mathrm{O}\left({ }^{3} P\right)+\mathrm{O}\left({ }^{3} P\right)+6.99 \mathrm{eV} \\
\mathrm{O}\left({ }^{3} P\right)+\mathrm{O}\left({ }^{1} D\right)+5.02 \mathrm{eV} \\
\mathrm{O}\left({ }^{1} D\right)+\mathrm{O}\left({ }^{1} D\right)+3.06 \mathrm{eV} \\
\mathrm{O}\left({ }^{1} D\right)+\mathrm{O}\left({ }^{1} S\right)+0.84 \mathrm{eV}
\end{array}\right.
$$

Oxygen atoms are formed in the ${ }^{3} \mathrm{P},{ }^{1} \mathrm{D}$, and ${ }^{1} \mathrm{~S}$ states and with the excess energies for each of channels equal to the half the energy release. The energy releases were calculated under the assumption that ions are in the ground vibrational state [Guberman, 1997; Fox, 2005]. Branching ratios for the channels (1) are taken as 0.22:0.42:0.31:0.05, based on the storage ring experiments by Kella et al. [1997].

[8] The process of precipitation of auroral electrons which causes excitation, dissociation, and ionization of atmospheric species has been added to this set of exothermic chemistry. In particular, dissociation and dissociative ionization of molecular oxygen can be considered as a source of suprathermal oxygen atoms:

$$
\begin{gathered}
O_{2}+e_{a} \rightarrow O\left({ }^{3} P\right)+O\left({ }^{3} P,{ }^{1} D,{ }^{1} S\right)+e_{a}+\Delta E_{O 2}^{d i s} \\
O_{2}+e_{a} \rightarrow O^{+}\left({ }^{4} S\right)+O\left({ }^{3} P,{ }^{1} D,{ }^{1} S\right)+e+e_{a}+\Delta E_{O 2}^{d i s-i},
\end{gathered}
$$

which are formed in the ground ${ }^{3} \mathrm{P}$ and electronically excited ${ }^{1} \mathrm{D}$, and ${ }^{1} \mathrm{~S}$ states, and with an excess of kinetic energy up to a few electron volts $(\mathrm{eV})$. The distribution of excess kinetic energy of $\mathrm{O}$ atoms in the electron impact dissociation $\left(\Delta E_{O 2}^{\text {dis }}\right)$ and dissociative ionization $\left(\Delta E_{O 2}^{\text {dis- }}{ }^{l}\right)$ of $\mathrm{O}_{2}$ molecules was estimated both from measurements [Cosby, 1993] and calculations [Van Zyl and Stephen, 1994]. Cross sections for these dissociation processes were taken from Cosby [1993] and Van Zyl and Stephen [1994].

\subsection{Auroral Proton/Hydrogen Precipitation}

[9] Interactions of precipitating energetic protons of magnetospheric origin with the main atmospheric constituents include momentum and energy transfer in elastic and inelastic collisions, ionization of target atmospheric molecules/ atoms, charge transfer and electron capture collisions. Energetic $\mathrm{H}$ atoms produced by proton impact further collide with the main atmosphere constituents, transferring their momentum and kinetic energy to atmospheric particles by elastic and inelastic collisions, ionization and stripping processes. The collisional processes describing the penetration of energetic $\mathrm{H}^{+} / \mathrm{H}$ into ambient atmosphere can be written as

$$
H^{+}(H)+M \rightarrow\left\{\begin{array}{c}
H_{f^{\prime}}^{+}\left(H_{f^{\prime}}\right)+M^{*} \\
H_{f^{\prime}}^{+}\left(H_{f^{\prime}}\right)+M^{+}+e \\
H_{f^{\prime}}\left(H_{f^{\prime}}^{+}\right)+M^{+}(M)+e
\end{array} .\right.
$$

Here, $\mathrm{M}$ denotes the major atmospheric constituents $\mathrm{O}_{2}, \mathrm{~N}_{2}$, and $\mathrm{O}$. Secondary fast $\mathrm{H}_{\mathrm{f}}$ atoms and $\mathrm{H}_{\mathrm{f}}{ }^{+}$protons produced by momentum transfer and stripping reactions recycle the reaction set given above. Consequently, the interaction of the precipitating protons with the main neutral thermospheric constituents must be considered as a cascade process producing a growing set of translationally and internally excited particles $\mathrm{M}^{*}$ of the ambient atmospheric gas. This process is known as the ion-induced atmospheric sputtering [Johnson, 1994].

[10] To analyze the penetration of energetic $\mathrm{H}^{+} / \mathrm{H}$ into the auroral atmospheric gas, we use kinetic Boltzmann equations [Gérard et al., 2000]. These coupled equations take 
into account both scattering and transport of the high-energy $\mathrm{H}^{+} / \mathrm{H}$ flux in elastic, inelastic, ionization, and charge transfer collisions with the ambient atmospheric gas. One of the consequences of the penetration of a high-energy $\mathrm{H}^{+} / \mathrm{H}$ flux into the upper atmosphere is the production of suprathermal oxygen atoms $\mathrm{O}_{h}$ by momentum transfer in elastic and inelastic collisions of the auroral $\mathrm{H}^{+} / \mathrm{H}$ beam with atmospheric oxygen $\mathrm{O}_{\text {th }}$ :

$$
H^{+}(H)+O_{t h} \rightarrow H^{+}(H)+O_{h}
$$

There is another possible channel of hot oxygen production due to the precipitation of high-energy $\mathrm{H}^{+} / \mathrm{H}$ fluxdissociative charge exchange of $\mathrm{O}_{2}$ with energetic protons. Recently, Luna et al. [2005] measured the cross sections and energy spectra of dissociation fragments in the dissociative charge exchange of $\mathrm{O}_{2}$ by fast $\mathrm{H}^{+}$over energy range $10-100 \mathrm{keV}$. They found that fragments in the dissociative charge exchange channel are formed with excess kinetic energies up to a few eV. Probably this source of hot oxygen is a minor one for the Earth's polar atmosphere, because the dissociative charge exchange of $\mathrm{O}_{2}$ by fast $\mathrm{H}^{+}$takes place in the relatively dense thermosphere where the $\mathrm{H}^{+} / \mathrm{H}$ flux is strongly depleted over the high-energy range. Upward moving hot oxygen atoms populate the corona and the fraction of their population with energies higher than energy of gravitational bounding forms the nonthermal escape flux.

[11] As mentioned before there are additional sources of energetic oxygen atoms such as charge exchange with the energetized ionospheric ions (neutral polar wind), and precipitation of high-energy oxygen ions from the ring current. In this study we do not consider the neutral polar wind because it mainly results in the formation of atomic hydrogen outflow flux [Gardner and Schunk, 2004]. Oxygen ion precipitation from the ring current is usually observed for very disturbed conditions such as the extremely strong magnetic storm on Bastille Day of 14 July 2000 [Wilson and Moore, 2005]. This source of hot oxygen was studied by Bisikalo et al. [1995]. Here we omit this source since we are interested in the comparison of hot oxygen sources in quiet to moderate magnetic conditions.

\section{Model for the Hot Oxygen Distribution Function}

[12] The fresh suprathermal oxygen atoms loose their excess kinetic energy in collisions with other atmospheric particles and are distributed in the transition region between the thermosphere and the exosphere [Shematovich et al., 1994, 1999, 2005]. Their kinetics and transport is described by the kinetic Boltzmann equation

$$
\mathbf{v} \frac{\partial}{\partial \mathbf{r}} f_{O h}+\mathbf{s} \frac{\partial}{\partial \mathbf{v}} f_{O h}=Q_{O h}(\mathbf{v})+\sum_{M=O, N_{2}, O_{2}} J_{m t}\left(f_{O h}, f_{M}\right),
$$

where $f_{O h}(\mathbf{r}, \mathbf{v})$, and $f_{M}(\mathbf{r}, \mathbf{v})$ are the velocity distribution functions for hot oxygen atoms, and components of ambient gas, respectively. The left side of the kinetic equation describes the transport of suprathermal oxygen in the planetary gravitational field $\mathbf{s}$. In the right-hand side of the kinetic equation the $\mathrm{Q}_{\mathrm{Oh}}$ term describes the formation rate of suprathermal oxygen atoms in the sputtering and chemical sources (1)-(4). The elastic and inelastic scattering terms $\mathrm{J}_{\mathrm{mt}}$ for hot oxygen collisions with ambient atmospheric species are written in a standard form [Shematovich et al., 1994]. It is assumed that the ambient atmospheric gas is characterized by local Maxwellian velocity distribution functions.

[13] The Direct Simulation Monte Carlo (DSMC) method is an efficient tool to study such complex kinetic systems in the stochastic approximation [Shematovich et al., 1994; Bisikalo et al., 1995; Gérard et al., 2000]. The details of the algorithmic realization of the numerical model were given earlier [Shematovich et al., 1994, 1999; Bisikalo et al., 1995]. The essence of the DSMC method is to generate a sample of paths for the state of the physical system under study, the hot oxygen collisions and transport in the transition region of the polar upper atmosphere in this case. Therefore, during the numerical realization of the kinetic model of the proton aurora, statistics for collisional processes forming suprathermal oxygen is accumulated and it provides the source function $\mathrm{Q}_{\mathrm{Oh}}$ due to the process (4). The GLOW model [Solomon et al., 1988] in the implementation by Hubert et al. [2001] was used to calculate the hot oxygen production rates due to the auroral electron precipitation and exothermic chemistry. The kinetic energy distribution of the nascent suprathermal $\mathrm{O}$ atoms was determined by the energy releases of the exothermic reactions under study: dissociative recombination of molecular oxygen ions (equation (1)), $\mathrm{O}_{2}$ electron impact dissociation (equations (2) and (3)), and the set of exothermic chemical reactions [Richards et al., 1994; Hickey et al., 1995; Gérard et al., 1995]. For dissociative recombination and chemical reactions the additional broadening of the initial energy distribution functions of the reaction products was also taken into account as by Shematovich et al. [1999]. These initial energy distributions of the reaction products together with production rates provide the total source function of the nascent hot $\mathrm{O}$ atoms due to the auroral electron precipitation and exothermic chemistry. Further, the source functions are used as an input into the stochastic model (5) of hot oxygen thermalization and transport in the transition region. The results of the hot oxygen model are the steady state energy distribution functions of oxygen atoms and the energy spectra of the upward fluxes of hot oxygen atoms at the upper boundary of the transition region under study.

[14] The thermalization rate of hot oxygen is determined by the collisional cross sections with the ambient neutral atmosphere. A key aspect of this model is the stochastic treatment of the scattering angle distribution in elastic and inelastic collisions of hot oxygen with ambient atmospheric gas. This effect influences both the pitch angle redistribution and the energy degradation through losses of energy in the momentum transfer collisions that are proportional to sine of the scattering angle. Recent detailed calculations of cross sections for elastic and inelastic collisions between suprathermal oxygen and atomic oxygen [Kharchenko et al., 2000], and molecular nitrogen [Balakrishnan et al., 1998a] were adopted. To take into account the scattering angle distribution, we used the calculated differential cross sections for O - O from Kharchenko et al. [2000], and for $\mathrm{O}-\mathrm{N}_{2}$ collisions we adopted the values calculated for 

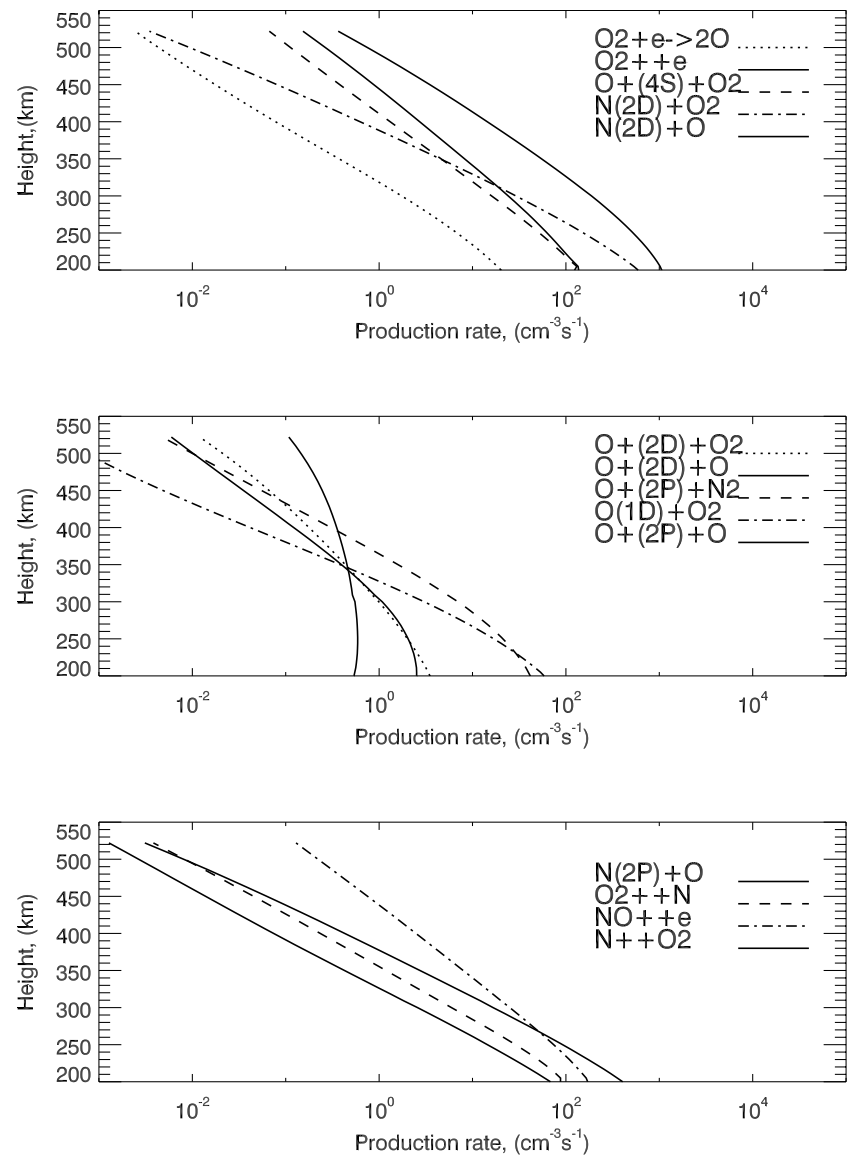

Figure 1. Auroral sources of energetic atomic oxygen due to the electron precipitation and exothermic chemistry in the auroral oval.

$\mathrm{N}-\mathrm{N}_{2}$ by Balakrishnan et al. [1998b]. The calculations were made for energies up to a few $\mathrm{eV}$, and we extrapolated the cross sections to higher collision energies with a (energy) $)^{-1 / 2}$ dependence according to the discussion by Gérard et al. [2000].

\section{Results of Numerical Simulations}

[15] Kinetics and transport of hot oxygen was considered in an atmospheric region with a lower boundary set at $80 \mathrm{~km}$ where hot atoms are efficiently thermalized and with an upper boundary at $700 \mathrm{~km}$ where the atmospheric gas flow is practically collisionless. The region of the atmosphere under study was divided into 49 radial cells. The altitudedependent cell size was chosen so that it is equal to or smaller than the local free path length for hot oxygen.

[16] To study the formation of energetic oxygen under auroral conditions, the two characteristic cases were considered: (1) nighttime polar oval when the electron precipitation is the dominant energy input and (2) daytime cusp when the proton precipitation is close to the electron energy input.

\subsection{Electron Aurora}

[17] As a first example, we consider the formation of hot oxygen atoms in the nighttime polar oval on 16 June 2000.
For this date we used the following input parameters, solar activity level of $\mathrm{F}_{10.7}=198$, geomagnetic activity level of $A p=5$. The latitude was selected $70^{\circ}$ and longitude was taken $0^{\circ}$. This corresponds to magnetic local and universal times equal to 0000 . The altitude distributions of the main neutral species, $\mathrm{O}_{2}, \mathrm{~N}_{2}$, and $\mathrm{O}$, and their temperature were calculated with the MSISE-90 reference model [Hedin, 1991]. The parameters of electron and proton precipitation at these conditions were taken from the Hardy et al. models [Hardy et al. 1985, 1989]. These empirical mean energy models were constructed from in situ data obtained from $\sim 27,000$ polar passes by DMSP spacecraft. Statistical maps of the mean particle energy, number flux, and energy flux were provided for electrons and protons for several different magnetic activity levels measured by $\mathrm{Kp}$ index. For our selected geophysical input parameters the Hardy et al. models give the following characteristics for the precipitating fluxes:

[18] 1. For electrons, number flux is $1.13 \times 10^{8} \mathrm{~cm}^{-2} \mathrm{~s}^{-1}$ and energy flux is $\mathrm{Q}_{0}=1.005 \mathrm{erg} \mathrm{cm} \mathrm{cm}^{-2} \mathrm{~s}^{-1}$. These values correspond to a mean energy $\overrightarrow{\mathrm{E}}=1.05 \mathrm{keV}$, and for the assumed Maxwellian distribution function, it will result in a characteristic energy $\mathrm{E}_{0}=0.52 \mathrm{keV}$.

[19] 2. For protons, number flux is $1.59 \times 10^{6} \mathrm{~cm}^{-2} \mathrm{~s}^{-1}$ and energy flux is $\mathrm{Q}_{0}=0.135 \mathrm{erg} \mathrm{cm}^{-2} \mathrm{~s}^{-1}$, corresponding to mean energy $\overline{\mathrm{E}}=8.44 \mathrm{keV}$, and for the assumed kappa distribution function with kappa $=3.5$, it will result in characteristic energy $\mathrm{E}_{0}=1.81 \mathrm{keV}$. The energy input to the polar upper atmosphere in this region is dominated by the electron precipitation. The energy input from proton precipitation is less than the electron distribution but nevertheless significant.

[20] The source function $\mathrm{Q}_{\mathrm{Oh}}$ of fresh hot oxygen atoms due to the proton precipitation was calculated by the proton auroral code for a precipitation with the mentioned above parameters. The fresh hot $\mathrm{O}$ atoms formed due to the momentum transfer in collisions with the incident $\mathrm{H}+/ \mathrm{H}$ flux are distributed by the energy up to $100 \mathrm{eV}$. Electron precipitation in the nighttime polar oval was calculated with an initial Maxwellian distribution with the adopted parameters, and the fresh hot $\mathrm{O}$ atoms resulting from electron auroral precipitation and induced chemistry are distributed with energies up to $5 \mathrm{eV}$. The rates of auroral sources of energetic atomic oxygen due to the electron precipitation and exothermic chemistry in the auroral oval are shown in Figure 1. The production rates of the fresh suprathermal oxygen atoms formed in processes (1)-(3) were calculated using the GLOW model [Solomon et al., 1988; Hubert et al., 2001] with the above mentioned updates of cross sections and branching ratios. It is seen that there is an significant input of the following reactions into the production of nascent hot oxygen atoms due to the electron precipitation

$$
\begin{gathered}
N\left({ }^{2} D\right)+O \rightarrow N\left({ }^{4} S\right)+O_{h}, \\
N\left({ }^{2} D\right)+O_{2} \rightarrow N O+O_{h}, \\
N^{+}+O_{2} \rightarrow N O^{+}+O_{h},
\end{gathered}
$$



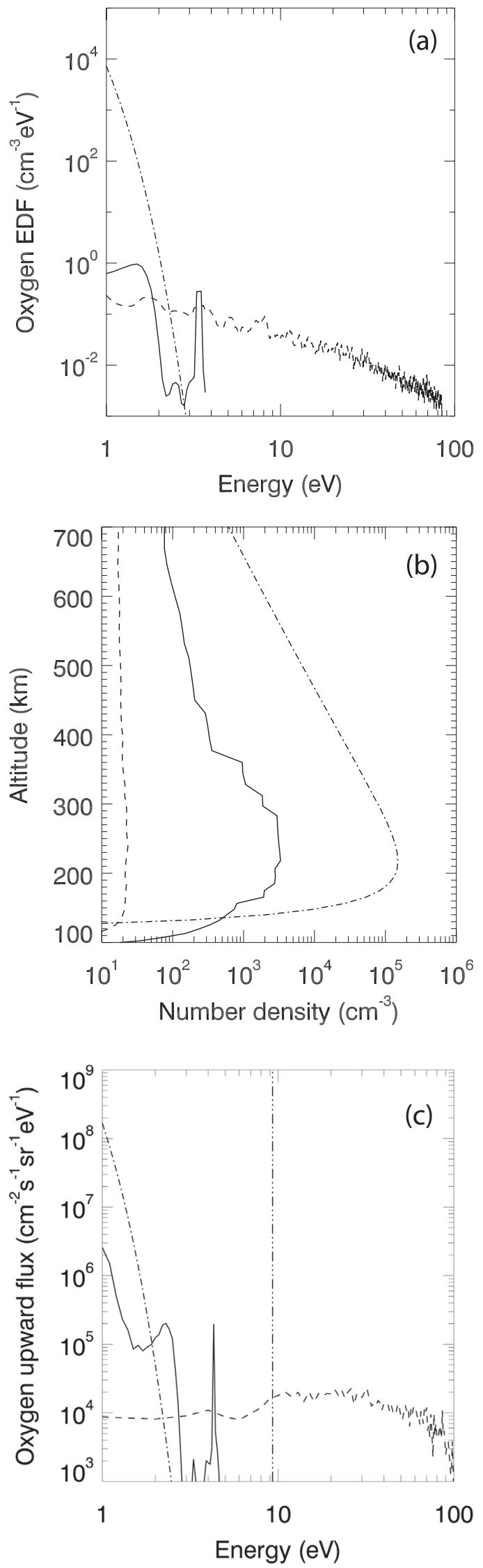

together with the dissociative recombination (1). Reaction (6c) results in the formation of $\mathrm{O}_{\mathrm{h}}$ atoms near the peak value of $4.35 \mathrm{eV}$. Other high-rate reactions $(6 \mathrm{a})$ and $(6 \mathrm{~b})$ result in the formation of fresh $\mathrm{O}$ atoms with energies of $1.11 \mathrm{eV}$ and $2.45 \mathrm{eV}$, respectively. Hot $\mathrm{O}$ atoms are formed with an energy spectrum extending up to $4 \mathrm{eV}$ in the dissociative recombination (1). Therefore these hot oxygen atoms mainly populate the very near-Earth region of the hot geocorona [Shematovich et al., 1994; Bisikalo et al., 1995; Gérard et al., 1995].

[21] The suprathermal oxygen atoms of both thermal and nonthermal origin populate the oxygen geocorona [Shematovich et al., 1994, 1999; Bisikalo et al., 1995]. The fraction of hot oxygen atoms from the region of suprathermal energies in the thermal Maxwellian distribution can be easily calculated using the reference empirical model MSISE-90 for the ambient atmosphere. Therefore the main problem is to calculate the distribution of hot oxygen of nonthermal origin.

[22] The energy distribution functions for suprathermal oxygen atoms were calculated and are shown in Figure $2 \mathrm{a}$ at $700 \mathrm{~km}$, above which the oxygen upward flux becomes practically collisionless. Here the distribution of hot $\mathrm{O}$ atoms originating from the ambient thermal atmospheric gas corresponds to the suprathermal tail of the Maxwellian distribution (for an exospheric temperature $\mathrm{T}=1150 \mathrm{~K}$ ) and is indicated by the dot-dashed line. The distributions of hot oxygen atoms of nonthermal origin are shown separately for electron (solid line) and proton (dashed line) precipitation. It is necessary to point out again that only energetic oxygen atoms with kinetic energies higher $1 \mathrm{eV}$ (i.e., the lower LENA observational threshold) were taken into account in the calculations presented here.

[23] It is seen that exothermic chemistry provides the main fraction of suprathermal atoms in the corona, while atmospheric sputtering is responsible for the formation of the escaping fraction of $\mathrm{O}$ atoms. The distributions of the number density and of upward fluxes of hot oxygen atoms in the nighttime polar oval are shown in Figures $2 \mathrm{~b}$ and 2c. These characteristics of hot oxygen were estimated from the energy distribution functions of suprathermal $\mathrm{O}$ atoms calculated by the stochastic Monte Carlo model. It is seen that the main contribution to the oxygen corona at exobase altitudes is due to the hot thermal atoms from the suprathermal tail of the local Maxwellian distribution. The nonthermal fractions are characterized by the larger scale

Figure 2. (a) Energy distribution functions at $700 \mathrm{~km}$ of thermal (dot-dashed line) and nonthermal oxygen calculated for electron (solid line) and proton (dashed line) precipitation in the nighttime auroral oval. (b) Altitude profiles of the population of oxygen atoms with a kinetic energy $>1 \mathrm{eV}$ for the thermal Maxwellian distribution (for an exospheric temperature $\mathrm{T}=1150 \mathrm{~K}$; dash-dotted line) and for nonthermal distributions corresponding to electron (solid line) and proton (dashed line) precipitation. (c) Energy spectra of the upward flux of hot oxygen at $700 \mathrm{~km}$. The vertical three-dot- dashed line shows the oxygen escape energy at $700 \mathrm{~km}$. Line styles are the same as in Figure 2a. 

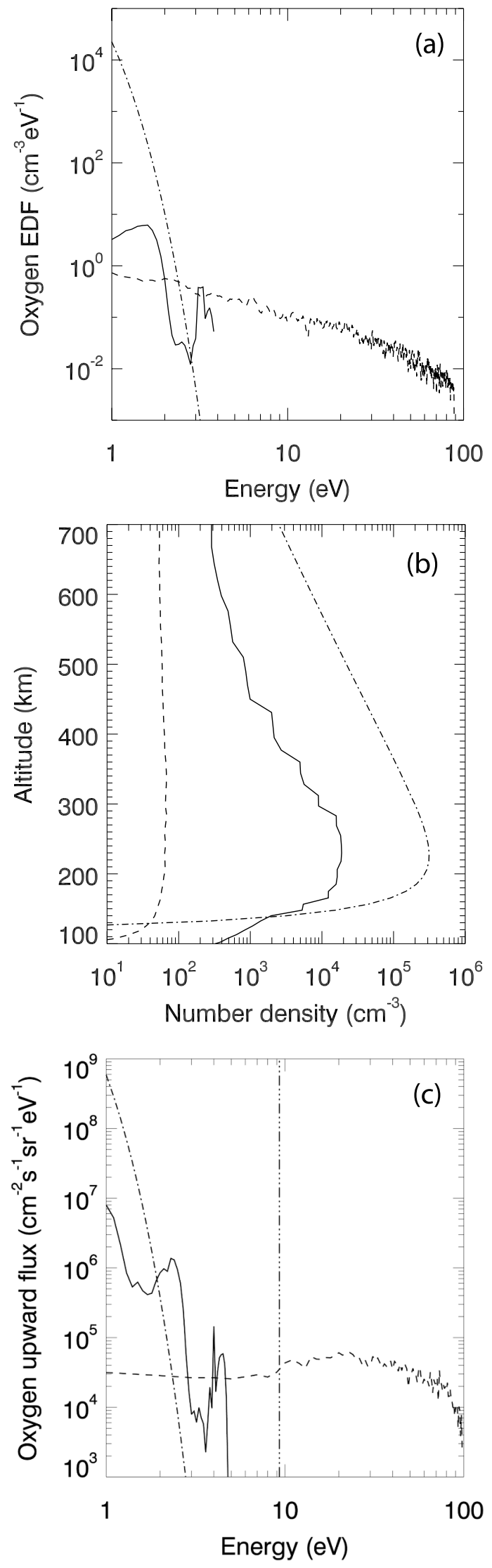

heights. Therefore the outer hot corona is preferentially populated by oxygen atoms of nonthermal origin.

\subsection{Proton Aurora}

[24] We now consider the formation of hot oxygen atoms in the daytime polar cusp on 16 June 2000. For this date we used the following input parameters, solar activity level of $\mathrm{F}_{10.7}=198$, geomagnetic activity level of $\mathrm{Ap}=5$. The latitude was selected $78^{\circ}$ and longitude was taken $0^{\circ}$. This corresponds to 1200 magnetic local time and the universal time was equal to $43,200 \mathrm{~s}$.

[25] The parameters of electron and proton precipitation under these conditions were taken from Hardy et al. model [Hardy et al., 1985, 1989] for given geophysical input parameters:

[26] 1. For electrons, number flux is $2.88 \times 10^{8} \mathrm{~cm}^{-2}$ $\mathrm{s}^{-1}$, and energy flux is $\mathrm{Q}_{0}=0.37 \mathrm{erg} \mathrm{cm}^{-2} \mathrm{~s}^{-1}$. These correspond to a mean energy $\overline{\mathrm{E}}=0.13 \mathrm{keV}$, and for the Maxwellian distribution function, it will result in characteristic energy $\mathrm{E}_{0}=0.065 \mathrm{keV}$.

[27] 3. For protons, number flux is $2.96 \times 10^{6} \mathrm{~cm}^{-2} \mathrm{~s}^{-1}$, and energy flux is $\mathrm{Q}_{0}=0.11 \mathrm{erg} \mathrm{cm} \mathrm{cm}^{-2} \mathrm{~s}^{-1}$. The mean energy is $\overline{\mathrm{E}}=3.51 \mathrm{keV}$, and, for the kappa distribution function with a kappa equal to 3.5 , it will result in characteristic energy $\mathrm{E}_{0}=0.75 \mathrm{keV}$.

[28] The energy distribution functions for suparthermal oxygen atoms at $700 \mathrm{~km}$ for the daytime cusp are shown in Figure 3a. The distributions of the number density and of upward fluxes of hot oxygen atoms in the daytime polar cusp are shown in Figures $3 \mathrm{~b}$ and $3 \mathrm{c}$.

[29] From these calculations it is seen that the energetic $\mathrm{O}$ atoms from the suprathermal tail of the local Maxwellian distribution mainly populate the hot oxygen corona at exobase altitudes. For energies higher than a few $\mathrm{eV}$, nonthermal processes (exothermic chemistry and atmospheric sputtering) become dominant sources of hot atoms. Moreover, atmospheric sputtering by the precipitating protons also results in the formation of a flux of escaping energetic oxygen atoms. The upward fluxes of hot oxygen shown in Figures $2 \mathrm{c}$ and $3 \mathrm{c}$ can be responsible for the energetic neutrals of auroral origin seen by the LENA instrument in both perigee and postperigee images [Wilson et al., 2003; Wilson and Moore, 2005]. To visualize the polar atmosphere response to the precipitation of magnetospheric protons, we calculated the map of escaping oxygen atoms for the averaged proton parameters on 16 June 2000. The averaged proton number flux calculated with Hardy et al. [1989] is shown in Figure 4 (left), and the

Figure 3. (a) Energy distribution functions at $700 \mathrm{~km}$ of thermal (dot-dashed line) and nonthermal oxygen calculated for electron (solid line) and proton (dashed line) precipitation in the daytime auroral cusp. (b) Altitude profiles of the population of oxygen atoms with a kinetic energy $>1 \mathrm{eV}$ for the thermal Maxwellian distribution (for an exospheric temperature $\mathrm{T}=1150 \mathrm{~K}$; dash-dotted line) and for nonthermal distributions corresponding to electron (solid line) and proton (dashed line) precipitation. (c) Energy spectra of the upward flux of hot oxygen at $700 \mathrm{~km}$. The vertical three-dot- dashed line shows the oxygen escape energy at $700 \mathrm{~km}$. Line styles are the same as in Figure 3a. 
Proton flux number

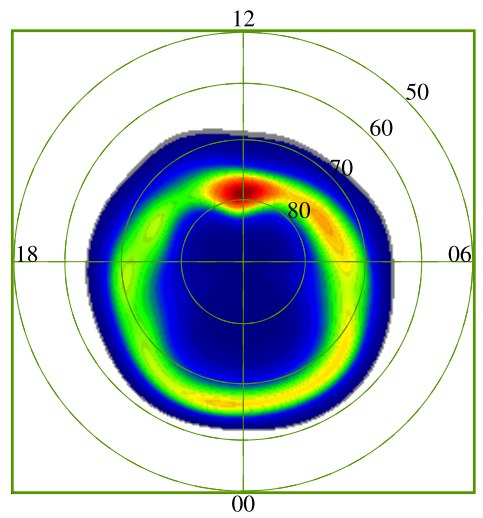

Oxygen escape flux

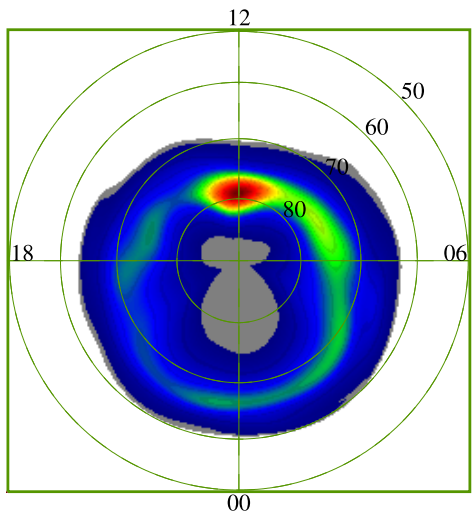

$10^{6} \mathrm{~cm}^{-2} \mathrm{~s}^{-1}$

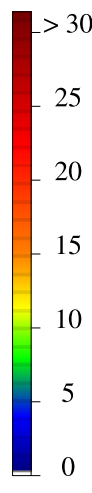

Figure 4. (left) Proton number flux from the Hardy et al. [1989] empirical model, using a Kp weighting evaluated for 16 June 2000. (right) Number flux of hot oxygen escaping from the polar atmosphere, calculated at $700 \mathrm{~km}$ for the proton flux on Figure 4 (left).

calculated escaping flux of oxygen atoms induced by these protons is displaced in the right panel. The mapping technique is same as the one used by Coumans et al. [2004]. It is seen that the cusp region is characterized by local oxygen escaping fluxes of $(10-30) \times 10^{6} \mathrm{~cm}^{-2} \mathrm{~s}^{-1}$. The total oxygen loss rate over the polar region is about $8 \times 10^{23}$ $\mathrm{s}^{-1}$, while the total oxygen loss rate over the cusp region is about $1 \times 10^{23} \mathrm{~s}^{-1}$. These estimates indicate that this source of neutral oxygen for the magnetosphere is comparable with the measurements reported by Seki et al. [2001] for the atmospheric loss of oxygen ions of about $10^{24}$ ions per second. This suggests that this mechanism may provide a substantial contribution to the magnetospheric oxygen population.

\section{Discussion and Conclusions}

[30] In this study we estimate the role of auroral sources (exothermic chemistry, electron and proton precipitation) in the formation of the hot oxygen corona in the polar upper atmosphere. It is found that these sources significantly contribute to the population of the hot oxygen geocorona and to the flux of upward moving oxygen atoms. It is important to point out that atmospheric sputtering by precipitating protons is the only process resulting in the formation of an escape flux of energetic oxygen atoms. Therefore it is expected that these auroral sources of suprathermal oxygen have signatures in the measurements of energetic neutrals in the near-Earth environment, such as those measured by the LENA instrument on board the IMAGE satellite.

[31] The exothermic chemistry induced by the electron precipitation and/or by the absorption of the solar UV radiation is operating continuously in the polar upper atmosphere and therefore it should be present in the observational data. Since the oxygen atoms originating from exothermic chemistry have low energies $(<5 \mathrm{eV})$, these energetic atoms can be only seen near the Earth when the LENA instrument looks in and near the ram direction. By contrast, atmospheric sputtering by magnetospheric protons as a source of energetic oxygen atoms will make much more variable contributions to the observations and it will also produce more energetic oxygen atoms that would be detectable farther away from the Earth and in directions away from ram. It is useful to point out that this source also produces the oxygen atoms with energies near $10 \mathrm{eV}$, which can be seen in low-latitude regions and far away from the ram direction. Wilson and Moore [2005] suggested, based on the analysis of the low-latitude perigee LENA data, that the instrument signal consists of low to medium energy (5$30 \mathrm{eV}$ ) oxygen atoms produced in and near the cusp region. Results of calculations confirmed this conclusion (see Figure 4). The more energetic $(>30 \mathrm{eV})$ fraction of oxygen atoms produced by the ion-induced atmospheric sputtering could be responsible for the energetic neutrals seen by the instrument far away from the cusp or oval region. The postperigee and high-latitude perigee LENA components again appear to be strongly associated with the escaping flux of oxygen atoms generated by atmospheric sputtering by precipitating magnetospheric protons.

[32] Acknowledgments. J. C. Gérard is supported by the Belgian Fund for Scientific Research (FNRS). This work was also supported by RFBR grant 05-02-17165 and funded by the Belgian National Fund for Collective Fundamental Research (FRFC grant 2.4517.02) and by the PRODEX programme of the European Space Agency (ESA).

[33] Wolfgang Baumjohann thanks Thomas Moore and Alexander Dalgarno for their assistance in evaluating this paper.

\section{References}

Balakrishnan, N., V. Kharchenko, and A. Dalgarno (1998a), Slowing of energetic $\mathrm{O}\left({ }^{3} \mathrm{P}\right)$ atoms in collisions with $\mathrm{N}_{2}, J$. Geophys. Res., 103, 23,393-23,398.

Balakrishnan, N., V. Kharchenko, and A. Dalgarno (1998b), Quantum mechanical and semiclassical studies of $\mathrm{N}+\mathrm{N}_{2}$ collisions and their application to thermalization of fast $\mathrm{N}$ atoms, J. Chem. Phys., 108, 943-949.

Bisikalo, D. V., V. I. Shematovich, and J.-C. Gérard (1995), Kinetic model of the formation of the hot oxygen geocorona. II. Influence of $\mathrm{O}^{+}$ion precipitation, J. Geophys. Res., 100, 3715-3720.

Cosby, P. C. (1993), Electron-impact dissociation of oxygen, J. Chem. Phys., 98, 9560-9569.

Coumans, V., J.-C. Gérard, B. Hubert, S. B. Mende, and S. W. H. Cowley (2004), Morphology and seasonal variations of global auroral proton precipitation observed by IMAGE-FUV, J. Geophys. Res., 109, A12205, doi:10.1029/2003JA010348.

Fox, J. L. (2005), Effects of dissociative recombination on the composition of planetary atmospheres, J. Phys. Conf. Ser., 4, 32-37.

Galand, M., and A. D. Richmond (1999), Magnetic mirroring in an incident proton beam, J. Geophys. Res., 104, 4447-4456. 
Gardner, L. C., and R. W. Schunk (2004), Neutral polar wind, J. Geophys. Res., 109, A05301, doi:10.1029/2003JA010291.

Gardner, L. C., and R. W. Schunk (2005), Global neutral polar wind model, J. Geophys. Res., 110, A10302, doi:10.1029/2005JA011029.

Gérard, J.-C., P. G. Richards, V. I. Shematovich, and D. V. Bisikalo (1995) The importance of new chemical sources for the hot oxygen geocorona, Geophys. Res. Lett., 22, 279-282.

Gérard, J.-C., B. Hubert, D. V. Bisikalo, and V. I. Shematovich (2000), A model of the Lyman- $\alpha$ line profile in the proton aurora, J. Geophys. Res. $105,15,795-15,806$.

Guberman, S. L. (1997), Mechanism for the green glow of the upper atmosphere, Science, 278, 1276-1278.

Hardy, D. A., M. S. Gussenhoven, and E. Holeman (1985), A statistical model of auroral electron precipitation, J. Geophys. Res., 90, 4229-4248.

Hardy, D. A., M. S. Gussenhoven, and D. Brautigam (1989), A statistical model of auroral ion precipitation, J. Geophys. Res., 94, 370-392.

Hedin, A. E. (1991), Extension of the MSIS thermosphere model into the middle and lower atmosphere, J. Geophys. Res., 96, 1159-1172.

Hickey, M. P., P. G. Richards, and D. G. Torr (1995), New sources for the hot oxygen geocorona: Solar cycle, seasonal, latitudinal, and diurna variations, J. Geophys. Res., 100, 17,377-17,388.

Hubert, B., J.-C. Gérard, D. V. Bisikalo, V. I. Shematovich, and S. C. Solomon (2001), The role of proton precipitation in the excitation of auroral FUV emissions, J. Geophys. Res., 106, 21,475-21,494.

Ishimoto, M., G. R. Romick, and C.-I. Meng (1992), Energy distribution of energetic $\mathrm{O}^{+}$precipitation into the atmosphere, J. Geophys. Res., 97 $8619-8629$.

Johnson, R. E. (1994), Plasma-induced sputtering of an atmosphere, Space Sci. Rev., 69, 215-253

Kella, D., L. Vejby-Christensen, P. J. Johnson, H. B. Pedersen, and H. Andersen (1997), The source of green light emission determined from a heavy-ion ring storage experiment, Science, 276, 1530-1533.

Kharchenko, V., A. Dalgarno, B. Zygelman, and J.-H. Yee (2000), Energy transfer in collisions of oxygen atoms in the terrestrial atmosphere, J. Geophys. Res., 105, 24,899-24,906.

Luna, H., C. McGrath, M. B. Shah, R. E. Johnson, M. Liu, C. J. Latimer, and E. C. Montenegro (2005), Dissociative charge exchange and ionization of $\mathrm{O}_{2}$ by fast $\mathrm{H}^{+}$and $\mathrm{O}^{+}$ions: Energetic ion interactions in Europa's oxygen atmosphere and neutral torus, Astrophys. J., 628, 1086-1096.
Moore, T. E., et al. (2000), The low energy neutral atom imager for IMAGE, in The IMAGE Mission, edited by J. L. Burch, pp. 155-195, Springer, New York.

Richards, P. G., M. P. Hickey, and D. G. Torr (1994), New sources for the hot oxygen geocorona, Geophys. Res. Lett., 21, 657-660.

Schunk, R. W., and A. F. Nagy (2000), Ionospheres, Cambridge Univ. Press, New York.

Seki, K., R. C. Elphic, M. Hirahara, T. Terasawa, and T. Mukai (2001), On atmospheric loss of oxygen ions from Earth through magnetospheric processes, Science, 291, 1939-1941.

Shematovich, V. I., D. V. Bisikalo, and J.-C. Gérard (1994), A kinetic model of the formation of the hot oxygen geocorona: 1. Quiet geomagnetic conditions, J. Geophys. Res., 99, 23,217-23,228.

Shematovich, V. I., J.-C. Gérard, D. V. Bisikalo, and B. Hubert (1999), Thermalization of $\mathrm{O}\left({ }^{1} \mathrm{D}\right)$ atoms in the thermosphere, J. Geophys. Res., 104, 4287-4295.

Shematovich, V. I., D. V. Bisikalo, and J.-C. Gérard (2005), An auroral source of hot oxygen in the geocorona, Geophys. Res. Lett., 32, L02105, doi:10.1029/2004GL021912.

Solomon, S. C., P. B. Hays, and V. Abreu (1988), The auroral 6300 A emission: Observation and modeling, J. Geophys. Res., 93, 9867-9882.

Wilson, G. R., and T. E. Moore (2005), Origins and variation of terrestrial energetic neutral atoms outflow, J. Geophys. Res., 110, A02207, doi:10.1029/2003JA010356.

Wilson, G. R., T. E. Moore, and M. R. Collier (2003), Low-energy neutral atoms observed near the Earth, J. Geophys. Res., 108(A4), 1142, doi:10.1029/2002JA009643.

Van Zyl, B., and T. M. Stephen (1994), Dissociative ionization of $\mathrm{H}_{2}, \mathrm{~N}_{2}$, and $\mathrm{O}_{2}$ by electron impact, Phys. Rev. A, 50, 3164-3173.

Yau, A., and M. André (1997), Source of ion outflow in the high latitude ionosphere, Space Sci. Rev., 80, 1-25.

D. V. Bisikalo and V. I. Shematovich, Institute of Astronomy RAS, 48 Pyatnitskaya Str., 119017 Moscow, Russia. (shematov@inasan.rssi.ru)

J.-C. Gérard, Laboratoire de Physique Atmosphérique et Planétaire, Institut d'Astrophysique et de Géophysique, Bat. B5c, Allee du 6 Aout, Université de Liège, Liège B-4000, Belgium. 